\title{
Is there an epigenetic component underlying the resistance of triple-negative breast cancers to Parp inhibitors?
}

\author{
Amanda Lovato, Lawrence Panasci and Michael Witcher* \\ The Departments of Oncology and Experimental Medicine, The Lady Davis Institute and Segal Cancer Centre of the Jewish General Hospital, McGill University \\ Montreal, QC, Canada
}

\section{Edited by:}

Gerald Batist, McGill University, Canada

Reviewed by:

William Douglas Figg, National Cancer Institute, USA

Brion William Murray, Pfizer Oncology Research Unit, USA

*Correspondence:

Michael Witcher, The Department of Oncology, The Lady Davis Institute and Segal Cancer Centre of the Jewish General Hospital, McGill University, Montreal, QC, Canada H3T 1 E2.

e-mail:michael.witcher@mcgill.ca
Poly(ADP-ribose) polymerase (Parp) is an enzyme responsible for catalyzing posttranslational modifications through the addition of poly(ADP-ribose) chains (known as PARylation). Modification by PARylation modulates numerous cellular processes including transcription, chromatin remodeling, apoptosis, and DNA damage repair. In particular, the role of Parp activation in response to DNA damage has been intensely studied. Tumors bearing mutations of the breast cancer susceptibility genes, Brca1/2, are prone to DNA breakages whose restoration into functional double-strand DNA is Parp dependent. This concept has been exploited therapeutically in Brca mutated breast and ovarian tumors, where acute sensitivity to Parp inhibitors is observed. Based on in vitro and clinical studies it remains unclear to what extent Parp inhibitors can be utilized beyond treating Brca mutated tumors. This review will focus on the often overlooked roles of PARylation in chromatin remodeling, epigenetics, and transcription to explain why some cancers may be unresponsive to Parp inhibition. We predict that understanding the impact of PARylation on gene expression will lead to alternative approaches to manipulate the Parp pathway for therapeutic benefit.

Keywords: Parp inhibitors, epigenetics, breast cancer, transcription factors, therapeutic resistance

\section{THE CLINICAL LANDSCAPE OF Parp INHIBITORS AS ANTI-CANCER THERAPEUTICS}

Breast cancer is an epidemic afflicting approximately one out of every nine women (Kurian et al., 2010). This heterogeneous disease is clinically stratified into four major subtypes; luminal A, luminal B, Her2+ [elevated Her2, Estrogen receptor (ER) negative], and triple-negative (Her2, ER, and progesterone receptor negative). However, current literature indicates further stratification using additional clinical markers, such as cytokeratins, Cyclin D1, and Claudin (Gusterson et al., 2005; Prat et al., 2010; Curtis et al., 2012), will provide more precise prognostic and predictive information. Patients whose tumors fall into the triple-negative category have the poorest clinical outcomes. While these aggressive tumors initially respond to chemotherapy, triple-negative patients are at high risk for metastatic recurrence and have poor overall survival (Di Cosimo and Baselga, 2010). Clearly, new therapeutic strategies are desperately needed to combat triple-negative tumors both at the time of onset and if necessary, at recurrence.

Approximately $10-20 \%$ of triple-negative tumors harbor a mutation in the Brcal/2 genes (Gonzalez-Angulo et al., 2011; Pern et al., 2012; Phuah et al., 2012). Brcal/2 proteins are an integral part of the homologous-recombination (HR) mediated DNA damage repair process. When mutated, the repair of double-strand breaks by HR is compromised. Mutations within the Brca genes are known to confer a $50-80 \%$ lifetime risk of breast cancer and a $20-40 \%$ lifetime risk of ovarian cancer for female carriers (Ford et al., 1998). An exciting new development in the field of cancer therapy is the exploitation of this defect using Poly(ADP-ribose) polymerase (Parp) inhibitors to generate synthetic lethality. Parp inhibitors have been used successfully to achieve clinical responses in patients with Brca mutated breast or ovarian cancers (Fong et al., 2009; Audeh et al., 2010; Tutt et al., 2010). Notably, the adverse effects of Parp inhibitors seen in clinical trials are quite mild with few signs that non-tumorigenic tissue is targeted by these drugs.

The role of Parp in the DNA damage repair response is multifaceted and already well-reviewed (Aly and Ganesan, 2011; Helleday, 2011). It is generally suggested throughout the scientific literature that Parp inhibitors generate an accumulation of single-strand DNA breaks in Brca mutated cells, which are subsequently processed to double-strand breaks during replication. However, the precise mechanism whereby Brca mutations and Parp inhibition combine to create a synthetic lethal effect remains somewhat controversial, with multiple mechanisms proposed (Helleday, 2011). Nonetheless, it seems clear that Parp inhibitors lead to stalled replication forks and an accumulation of DNA damage, particularly cytotoxic in cells with a mutant Brca background.

The clinical efficacy of Parp inhibitors in triple-negative breast tumors bearing Brca mutations has sparked interest to initiate new clinical trials in triple-negative patients having wild-type Brca with the expectation that combining chemotherapy with Parp inhibitors will enhance the outcomes of currently utilized therapies. While the results from a few phase one trials are encouraging, to date, most have been met with limited success. Preliminary data from a cohort of 86 triple-negative patients cotreated with the Parp inhibitor Bsi-201 (iniparib) and gemcitabine showed improved overall survival (O'Shaughnessy et al., 2009). Similarly, co-treatment of a 123 triple-negative patient cohort 
with gemcitabine and iniparib improved overall survival from 7.7 to 12.3 months (O'Shaughnessy et al., 2011). But a larger phase III trial involving 519 women showed co-treatment of cytotoxic agents with the Parp inhibitor Iniparib was associated with disease progression in most cases (O'Shaughnessy, 2011). However, results from these trials should be taken with caution as recent data suggests Parp is not the primary target of iniparib (Liu et al., 2012). Another phase II trial looking at the efficacy of the Parp inhibitor olaparib in breast cancer patients failed to show significant clinical responses (Gelmon et al., 2011). Encouragingly, in this same study, a cohort of patients with ovarian cancers did demonstrate partial responses to olaparib regardless of Brca status.

Overall, the early indicators from trials involving Parp inhibitors for triple-negative breast cancer show partial, but not complete responses. Encouragingly, there are clearly patients who do respond to these therapies. Recent research has identified a subset of triple-negative tumors that have an increased likelihood to respond to Parp inhibition. Defective proteins in the HR repair system, or epigenetically silenced Brca, amongst other defects, contribute to a molecular pathology that is not unlike tumors bearing Brca mutations. These tumor properties are defined as having "Brcaness" or being "Brca-like" (Turner et al., 2004; Ratner et al., 2012). This concept has led to the hypothesis that tumors with features of Brcaness may respond to Parp inhibition. The concept of Brcaness has been used in retrospective study to predict response to platinum-based therapies in 8 of 10 patients (Konstantinopoulos et al., 2010). But a recent study analyzing data from 101 patients receiving adjuvant cyclophosphamide-based chemotherapy showed Brcaness could not predict differences in patient survival (Oonk et al., 2012). However, it still remains to be determined if pathological features of Brcaness may be a more powerful predictor of sensitivity to Parp inhibitors than conventional chemotherapy.

Current literature suggests several hypotheses to predict sensitivity to Parp inhibition in subsets of triple-negative tumors, but there is a lack of evidence from in vitro and mouse studies suggesting that established triple-negative cell lines are sensitive to clinically relevant Parp inhibitors or Parp-1 knock down. In fact, unpublished data from our lab and another recent report show very high concentrations of commonly used Parp inhibitors are needed to suppress the growth of triple-negative cell lines in vitro (Chuang et al., 2012). The micromolar concentrations of inhibitors needed to suppress proliferation is likely well beyond those required to block Parp activity (Bryant et al., 2005; Liu et al., 2008), and may reflect secondary effects of these inhibitors.

Beyond breast cancer, early phase clinical trials with Parp inhibitors in combination with standard chemotherapy have been met with either partial responses, or a lack of clinically relevant responses in multiple types of solid tumors (Plummer et al., 2008; Khan et al., 2011; Kummar et al., 2011, 2012; Rajan et al., 2012). We propose that activities of Parp, being targeted by inhibitors beyond those in the DNA damage repair process, account for their limited success in a wild-type Brca background (Figure 1). Specifically, we hypothesize that targeting Parp will impair its role in regulating the expression of tumor suppressor genes, thereby generating unwanted consequences. However, we further propose that understanding the mechanisms whereby Parp activates transcription may be used to predict new, more potent therapeutic approaches.

\section{POLY(ADP)POLYMERASES, MORE THAN DNA REPAIR ENZYMES}

The Parp family of enzymes encompasses multiple proteins (up to 17) of varying degrees of homology (with the main conservation residing in the Parp catalytic domain), all of whom use $\mathrm{NAD}+$ as a substrate to catalyze the addition of ADP-ribose moieties onto target proteins (Kim et al., 2005). Among the Parp proteins, only Parp-1 and Parp-2 build "poly" ADP-ribose polymers. The other family members, including Parp-3, are capable of adding only a monomeric ADP-ribose to proteins. Of Parp-1 and 2 , Parp-1 has been more extensively studied and will therefore be the focus of this review. Under basal conditions, Parp-1 is active, but modifies relatively few target proteins compared to conditions of cellular stress and after DNA damage (Gagne et al., 2008, 2012; Witcher and Emerson, 2009). Parp-1 recognizes and binds specific DNA secondary structures commonly associated with damaged DNA including single-strand DNA, double-strand breaks, and crossovers. Upon binding, its enzymatic activity is allosterically triggered (D’Amours et al., 1999; Kun et al., 2002). This particular mechanism of regulation allows localized activation of Parp-1 for targeted repair of DNA damage. However, numerous studies have also demonstrated that Parp-1 is recruited to chromatin, which acts as an on-switch for its enzymatic activity in the absence of DNA damaging agents (Poirier et al., 1982; Ding and Smulson, 1994; Kim et al., 2004; Lonskaya et al., 2005; Pinnola et al., 2007; Wacker et al., 2007).

Parp-1 associates with the chromatin of promoter regions in a significant proportion of actively transcribed genes throughout the genome (Krishnakumar and Kraus, 2010; Zhang et al., 2012) and copurifies biochemically with RNA Pol II (Slattery et al., 1983). Consistent with this, multiple studies utilizing Parp-1 depleted cells have substantiated an activating role for Parp-1 in gene regulation (Ziegler and Oei, 2001; Ogino et al., 2007; Krishnakumar et al., 2008; Okada et al., 2008; Frizzell et al., 2009).

How does Parp modulate gene expression? It does so through a multi-pronged approach, with individual actions cooperating to fine tune the transcriptional process (Figure 1). Parp-1 regulates transcription minimally through (1) the alteration of chromatin structure (2) the control of DNA and histone methylation status (3) the recruitment and maintenance of transcription factors to promoter regions, and (4) acting as a transcriptional coregulator.

\section{Parp-1 AS A REGULATOR OF TRANSCRIPTION}

First, direct PARylation of histones can lead to a loosening of chromatin conformation. PARylation of bulk nucleosomes in vitro leads to decondensation (Faraone-Mennella et al., 1993) and Parp activity is required for chromatin loosening at stress induced genes in Drosophila (Tulin and Spradling, 2003). Biochemically, the negative charge conferred by the PAR group onto histones promotes their release from the DNA due to charge repulsion. Such is the case for histone $\mathrm{H} 1$, a heterochromatin-promoting factor whose PARylation-dependent removal from chromatin serves to promote chromatin relaxation (Poirier et al., 1982; Huletsky et al., 1989). Interestingly, Parp-1 localization is inversely related 


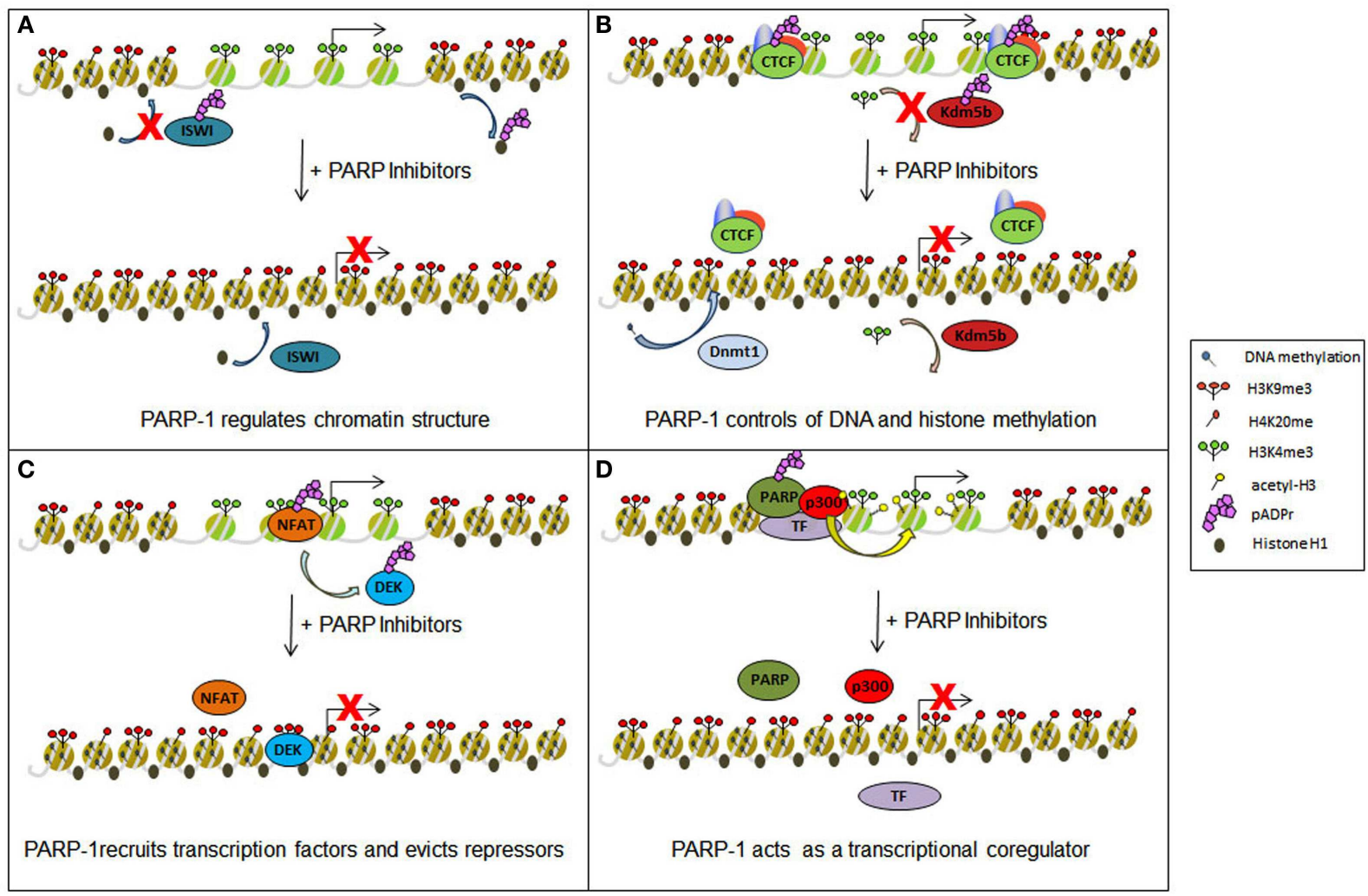

FIGURE 1 | Epigenetic and transcriptional impact of Parp inhibitors. Parp inhibitors may contribute to epigenetic and transcriptional deregulation in cells through several different mechanisms. (A) The drug-induced spread of heterochromatin may result from the release of the protein Iswi from inhibition, promoting histone $\mathrm{H} 1$ integration into chromatin, and through the prevention of histone $\mathrm{H} 1$ removal from chromatin by direct PARylation. (B) Chromatin boundaries normally maintained by PARylated Ctcf may be disrupted and demethylation of $\mathrm{H} 3 \mathrm{~K} 4 \mathrm{me} 3$ by $\mathrm{Kdm} 5 \mathrm{~b}$ restored with the use of
Parp inhibitors. In both incidencies, transcriptional inhibition will ensue. Such drugs may also act to restore Dnmt1 methylation of DNA, further promoting gene silencing. (C) Parp inhibition can disrupt protein: DNA interactions, preventing the maintenance of certain trans-activating factors (e.g., Nfat) at transcription start sites while also causing the retention of some repressor proteins (e.g., Dek). (D) Gene activation may also be negatively regulated by preventing Parp from acting as a transcriptional coregulator and obstructing the recruitment of such proteins as the histone acetyl transferase p300. to histone $\mathrm{H} 1$ binding throughout the genome and higher proportions of Parp-1:H1 proteins tend to indicate active promoters (Krishnakumar et al., 2008). Consistent with this, experiments using Parp-1 mutants and Parp inhibitors in Drosophila revealed more pronounced heterochromatin at the Heat shock protein 70 (Hsp70) locus (Tulin and Spradling, 2003). Likewise, an RNAi screen revealed Parp is necessary for nucleosome eviction from chromatin at the Hsp70 locus during the rapid gene induction response to heat shock (Petesch and Lis, 2008). Recently, it has been demonstrated that lipopolysaccharide-induced Parp activity displaces nucleosomes from target genes, thereby facilitating transcription, as trans-activating factors will not have to contend with the physical obstacle of dense nucleosomes at these promoters (Martinez-Zamudio and Ha, 2012). In sum, the ability of Parp-1 to remodel chromatin in a manner conducive to transcriptional activation strongly suggests that Parp-1 primarily acts as a potent activator of transcription. However, in some contexts, Parp-1 may promote a repressed chromatin conformation when enzymatically inactive, and a more loose structure upon activation (Wacker et al., 2007). At a subset of promoters, under unstimulated conditions, Parp-1 presents itself in a corepressor complex with nucleolin, nucleophosmin, and Hsp70. These repressive factors, however, are released upon signal activation of Parp-1, thus providing a mechanism for differential effects of Parp-1 on chromatin structure (Ju and Rosenfeld, 2006).

Countering the effects of PARylation on chromatin structure is poly(ADP-ribose) glycohydrolase (known as Parg). Parg catabolizes ADP-ribose polymers synthesized by Parp-1. This enzymatic activity has been demonstrated to impair Parp-mediated chromatin remodeling in vitro (Kim et al., 2004). Chromatin remodeling mediated by Parp-1 potentiates transcriptional activation by the ER (Kim et al., 2004) and Parg was shown to suppress estrogen-dependent transcription through blocking Parp activity. Further work is required to elucidate the in vivo action of Parg on chromatin structure.

In addition to histone $\mathrm{H} 1$ removal, Parp-1 configures chromatin through modification of proteins involved in remodeling and organizing chromatin structure. PARylation generally results in protein activation, but can also result in functional suppression of chromatin remodelers. For example, PARylation is inhibitory to the function of the repressive remodeling complex Iswi (Sala et al., 2008). Iswi is known to promote the association between $\mathrm{H} 1$ 
and DNA (Corona et al., 2007), thus illustrating a complementary mechanism by which PARylation results in a reduction of $\mathrm{H} 1$ binding to DNA.

Notably, Parp interaction with Brg-1 (SmarcA4), together with histone deacetylases (Hdacs), results in a repressive complex, inactivating the transcription of genes involved in cardiomyocyte differentiation through deacetylation of histones (Hang et al., 2010). Thus, in particular contexts, Parp-1 activity can relay transcriptionally repressive signals. Conversely, this same study showed that a Parp-1/Brg-1 complex devoid of Hdac could activate a separate subset of genes. It remains to be seen if the PARylation of Brg-1 leads to gene activation in other tissue types.

In addition, other chromatin remodelers are modified by Parp1 under conditions of cellular stress (Gagne et al., 2008, 2012). Proteomic studies have shown these to include TopoII $\alpha$, Brg-1, TopoII $\beta$, HmgA1, Chd1, Chd5, and Snf2L1. Clearly, Parp activation relays a signal that is having a profound effect on chromatin structure. However, the precise impact PARylation has on these proteins, and the subsequent effects on transcription of their target genes remains unknown.

Second, Parp activity regulates gene expression through control of epigenetic mechanisms including histone modification and DNA methylation. Parp covalently modifies the epigenetic regulatory protein CCCTC binding factor (Ctcf; Yu et al., 2004). Ctcf PARylation is important for its insulator activity, which functions to prevent enhancers and repressors from acting on distal promoters over long distances. Therefore, inhibition of Ctcf PARylation will result in altered regulation of target genes through the aberrant actions of distal regulatory regions.

Chromatin immunoprecipitation experiments and knockdown studies indicate Ctcf plays an import role in the maintenance of chromatin boundaries (Cuddapah et al., 2009; Witcher and Emerson, 2009). Repressive heterochromatin is the default state and, unless constrained, will spread passively throughout a chromosome (Talbert and Henikoff, 2006). Chromatin boundaries form a barrier prohibiting the spread of repressive chromatin. Interestingly, Ctcf PARylation has been linked to the maintenance of chromatin boundaries at tumor suppressor genes (Witcher and Emerson, 2009; Farrar et al., 2010).

This is supported by unpublished data from our lab showing Parp inhibitors lead to an accumulation of repressive histone modifications, such as $\mathrm{H} 3 \mathrm{~K} 27 \mathrm{me}^{3}$, at tumor suppressor genes. We have also published that Parp-1 inhibition through knockdown, or pharmacologic approaches, results in the transcriptional repression of the Rassf1a and p16 tumor suppressor genes (Witcher and Emerson, 2009). Based on this data, it is not unexpected that Parp inhibitors have been found to have transcriptionally repressive effects on tumor suppressor genes (Witcher and Emerson, 2009; Nguyen et al., 2011). However, it remains to be proven that these negative effects are mediated by disruption of Ctcf function.

Beyond modulating its role as a chromatin boundary protein, PARylation of Ctcf may act as a docking site for Dnmt1 binding (Zampieri et al., 2009, 2012). This interaction is thought to act a molecular sponge, prohibiting Dnmtl from methylating regions surrounding Ctcf binding sites. Elegant studies utilizing engineered mutations of endogenous Ctcf sites clearly show localized accumulation of DNA methylation when Ctcf binding is abolished (Pant et al., 2004; Davalos-Salas et al., 2011). However, it remains to be proven that the interaction between Ctcf and Dnmt1 is pivotal for the capacity of Ctcf to prevent DNA methylation. Nevertheless, this model again suggests that a loss of Ctcf PARylation brought about by Parp inhibitors would result in profound epigenetic changes and significant changes to gene expression throughout the genome. Supporting this model is at least one study showing that Parp inhibition does indeed result in widespread accumulation of DNA methylation (Reale et al., 2005).

In addition to epigenetic regulation through Ctcf, PARylation of other target proteins organize chromatin structure through coordinating the placement of histone modifications. This has been most clearly demonstrated for the histone demethylase $\mathrm{Kdm} 5 \mathrm{~b}$ (Krishnakumar and Kraus, 2010). Modification of Kdm5b by Parp-1 allows H3K4me3 to persist in the promoter regions of actively transcribed genes. $\mathrm{H} 3 \mathrm{~K} 4 \mathrm{me} 3$ is important for loading the PolII machinery at most, if not all, actively transcribed genes. PARylation of Kdm5b prevents active demethylation of this mark at $\mathrm{Kdm} 5 \mathrm{~b}$ target genes, thus promoting transcription. Knockdown of Parp-1 potently blocks transcription of Kdm5b target genes through this mechanism. Clearly, small molecule inhibitors or Parp-1 would be expected to disrupt transcription in a similar fashion.

Third, Parp activity controls the recruitment and maintenance of transcription factors to promoter regions. As stated above, classic experiments from the Roder lab demonstrate that Parpcopurifies biochemically with RNA PolII (Slattery et al., 1983). Follow up work showed Parp enhances the assembly of the preinitiation complex in vitro (Meisterernst et al., 1997). Surprisingly, Parp activity has been demonstrated to be necessary to retain Pol II at actively transcribed target genes (Zobeck et al., 2010). It is postulated that PAR polymers creates a scaffold that retains Pol II at gene loci. It is quite possible, but remains to be proven, that PAR scaffolds act to retain transactivators at target genes as well. The trans-activating factors Nfat, Klf8, and Tef-1 are associated with, and activated by PAR polymers (Butler and Ordahl, 1999; Olabisi et al., 2008; Lu et al., 2011). It is possible the mechanism lies in the retention of these factors at target genes by (ADP)ribose polymers.

In contrast to this model, PARylation of the transcriptional repressor Dek by Parp-1 serves to evict Dek from chromatin, ultimately promoting gene activation (Gamble and Fisher, 2007). Thus, blocking the actions of repressors serves as another mechanism through which Parp activates transcription.

Fourth, Parp-1 itself has also been described to act as a coregulator of transcription. It is recruited to genes via interaction with DNA binding factors. As a coregulator, Parp-1 can be integrated into complexes having stimulatory effects on transcription mediated by transcription factors such as NF- $\kappa \beta$ and AP-2 (Li et al., 2004). To complete the assembly of the NF- $\kappa \beta$ activating complex, Parp-1 is required for the integration of the histone acetyltransferase p300 (Hassa et al., 2003; Kaur et al., 2006), providing another link between Parp-1 and histone modification. Parp-1 is also a component of a coactivating complex responsible for driving nuclear hormone receptor-mediated transcription in response to estrogens and retinoids (Pavri et al., 2005; Ju et al., 2006). 
Docking with Parp-1 can also serve to enhanced phosphorylation of the associated transcription factor leading to heightened trans-activating capabilities. This has been demonstrated for BMyb in a cell cycle dependent fashion (Cervellera and Sala, 2000; Santilli et al., 2001) and Elk-1 in response to Erk-1 activation (Cohen-Armon et al., 2007). These data indicate Parp activity links intra and extracellular signaling events with gene induction.

Parp-1 knockdown studies show Parp activity also functions to repress a subset of genes (Frizzell et al., 2009). Consistent with this, PARylation of a number of transcription factors has been described to prevent their interaction with DNA. This has been described for Smads (after TGF $\beta$ stimulation; Lonn et al., 2010), p53, and Sp1 (Kumari et al., 1998; Malanga et al., 1998; Zaniolo et al., 2007). Inactivation of Smads by Parp following TGF $\beta$ signaling remains controversial as a more recent study found Parp necessary for Smad activation post-TGF $\beta$ exposure (Huang et al., 2011). It will be of great interest in the future to determine how Parp integrates signaling events such as TGF $\beta$, reactive oxygen species, and growth factors into coordinated transcriptional outputs.

Information from in vitro studies describing the inhibition of transcription factor binding to cognate sites by PARylation should be taken with caution. Without the constraints found in vivo, ADP-ribose polymers can be extended to enormous lengths in vitro (D'Amours et al., 1999; Mendoza-Alvarez et al., 2000). It is likely that the formation of such a network of polymers could impair binding to cognate DNA sites in vitro due to simple steric hindrance.

Overall, evidence suggests that Parp-1 plays primarily a stimulatory role on transcription, including activation of tumor suppressor genes and a more minor role in gene repression. Clearly there is overwhelming data demonstrating that Parp-1 participates in gene regulation at multiple levels, most prominently by coordinating transcription factor activity and organizing chromatin structure. It is imperative that these aspects of Parp-1 function be considered, along with its role in DNA damage repair if we are to extend the clinical use of Parp inhibitors to treat tumors beyond those bearing Brca mutations.

\section{CLINICAL MODULATION OF THE POLY(ADP)RIBOSE PATHWAY: FUTURE PERSPECTIVES}

The epigenetic, chromatin remodeling, and transcriptional regulatory roles of Parp-1 are necessary to activate a group of genes under basal conditions and another cohort in response to stimuli, such as cell stress. Therefore, inhibiting Parp-1 will potentially disrupt expression of a wide range of genes, including tumor suppressor genes, which may limit the benefits of Parp inhibitors in Brca wild-type patients.

That being said, can this pathway be targeted successfully to treat a broad range of tumors? In our opinion, yes, but we need to revisit our approach. First, it is clear that Parp-2 can compensate for Parp-1. Thus Parp inhibitors should be tested for their capacity to block the activity of both proteins before being considered for clinical trials. Such consideration might have prevented the failed clinical trials with iniparib, a drug initially described as a Parp inhibitor, but that has recently been proven to lack such activity (Patel et al., 2012).

Second, the concept of Brcaness needs to be more clearly defined using both genetic and epigenetic markers. It is probable that tumors with epigenetically silenced, as well as mutated, DNA repair genes will be sensitive to Parp inhibitors. This concept may also be employed to predict successful combinations of chemotherapeutics with Parp inhibitiors. Further, it is possible that epigenetic silencing of tumor suppressor genes that are Parp targets may negate any pro-proliferative effects of Parp, potentially rendering the cells sensitive to Parp inhibition. To date, in vitro models have been used to accurately predict tumor pathologies that are clinically sensitive to Parp inhibitors (Bryant et al., 2005; Donawho et al., 2007). Therefore, future testing of Brcaness models using in vitro systems will be an important stepping stone to make these models clinically relevant.

Finally, we propose that Parg represents an attractive therapeutic target (Figure 2). The understudied protein Parg catalyzes the counter-activity of Parp-1/2 by removing PARylation modifications from target proteins. Intriguingly, Parg has a

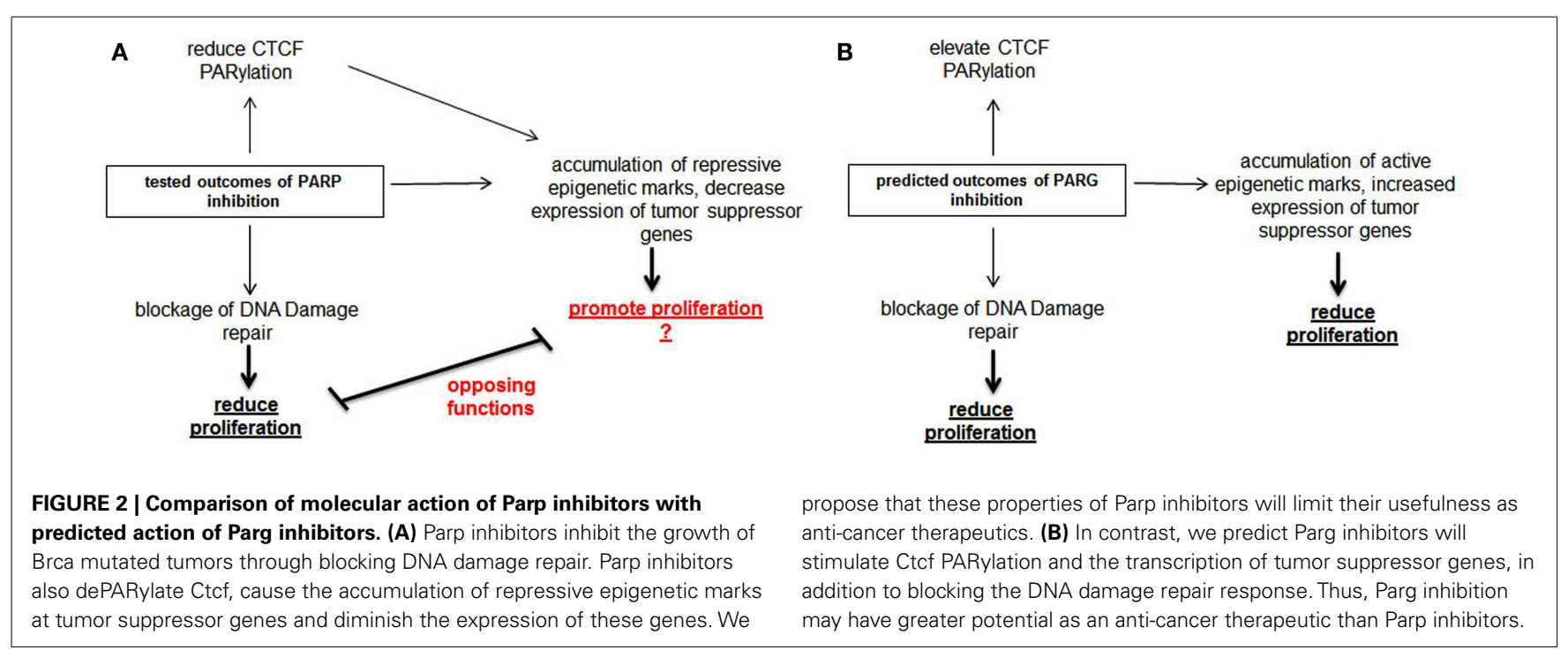


critical role in DNA damage repair, similar to Parp-1 (Fisher et al., 2007; Mortusewicz et al., 2011). Counter intuitively, while PARylation of target proteins is necessary to initiate the repair response to DNA damage, removal of these tags is essential for a complete DNA damage response. Thus, Parg inhibition would likely create a synthetic lethal situation in Brca defective cells in a similar manner as Parp inhibitors. Consistent with this concept, Brca mutated cells have been shown to be highly sensitive to an inhibitor of Parg (Fathers et al., 2012). Further, we now have exciting new data demonstrating Parg inhibition is a relevant approach to inhibit the proliferation of triple-negative breast cancer cell both in vitro and in vivo (manuscript in preparation).

Parp-1 is an important activator of transcription and probably plays an important role in promoting transcription of tumor suppress genes. Therefore, we postulate Parp inhibitors may actually have pro-oncogenic effects on some cell populations. But, what is the impact of inhibiting Parg on these same processes? While the overall impact of Parg on gene regulation remains

\section{REFERENCES}

Aly, A., and Ganesan, S. (2011). BRCA1, PARP, and 53BP1: conditional synthetic lethality and synthetic viability. J. Mol. Cell Biol. 3, 66-74.

Audeh, M. W., Carmichael, J., Penson, R. T., Friedlander, M., Powell, B., Bell-McGuinn, K. M., et al. (2010). Oral poly(ADP-ribose) polymerase inhibitor olaparib in patients with BRCA1 or BRCA2 mutations and recurrent ovarian cancer: a proof-of-concept trial. Lancet 376, 245-251.

Bryant, H. E., Schultz, N., Thomas, H. D., Parker, K. M., Flower, D., Lopez, E., et al. (2005). Specific killing of BRCA2-deficient tumours with inhibitors of poly(ADPribose) polymerase. Nature 434, 913-917.

Butler, A. J., and Ordahl, C. P. (1999). Poly(ADP-ribose) polymerase binds with transcription enhancer factor 1 to MCAT1 elements to regulate muscle-specific transcription. Mol. Cell. Biol. 19, 296-306.

Cervellera, M. N., and Sala, A. (2000). Poly(ADP-ribose) polymerase is a BMYB coactivator. J. Biol. Chem. 275, 10692-10696.

Chuang, H. C., Kapuriya, N., Kulp, S. K., Chen, C. S., and Shapiro, C. L. (2012). Differential anti-proliferative activities of poly(ADP-ribose) polymerase (PARP) inhibitors in triple-negative breast cancer cells. Breast Cancer Res. Treat. 134, 649-659.

Cohen-Armon, M., Visochek, L., Rozensal, D., Kalal, A., Geistrikh, I., Klein, R., et al. (2007). DNA-independent PARP-1 activation by phosphorylated ERK2 increases Elk1 activity: a link to histone acetylation. Mol. Cell 25, 297-308.
Corona, D. F., Siriaco, G., Armstrong, J. A., Snarskaya, N., McClymont, S. A., Scott, M. P., et al. (2007). ISWI regulates higher-order chromatin structure and histone $\mathrm{H} 1$ assembly in vivo. PLoS Biol. 5:e232. doi:10.1371/journal.pbio.0050232

Cuddapah, S., Jothi, R., Schones, D. E., Roh, T. Y., Cui, K., and Zhao, K. (2009). Global analysis of the insulator binding protein CTCF in chromatin barrier regions reveals demarcation of active and repressive domains. Genome Res. 19, 24-32.

Curtis, C., Shah, S. P., Chin, S. F., Turashvili, G., Rueda, O. M., Dunning, M. J., et al. (2012). The genomic and transcriptomic architecture of 2,000 breast tumours reveals novel subgroups. Nature 486, 346-352.

D’Amours, D., Desnoyers, S., D'Silva, I., and Poirier, G. G. (1999). Poly(ADPribosyl)ation reactions in the regulation of nuclear functions. Biochem. J. 342(Pt 2), 249-268.

Davalos-Salas, M., Furlan-Magaril, M., Gonzalez-Buendia, E., ValdesQuezada, C., Ayala-Ortega, E., and Recillas-Targa, F. (2011). Gain of DNA methylation is enhanced in the absence of CTCF at the human retinoblastoma gene doi:10.1186/1471-2407-11-232

Di Cosimo, S., and Baselga, J. (2010). Management of breast cancer with targeted agents: importance of heterogeneity. Nat. Rev. Clin. Oncol. 7, 139-147. [Corrected].

Ding, R., and Smulson, M. (1994). Depletion of nuclear poly(ADPribose) polymerase by antisense RNA expression: influences on genomic stability, chromatin organization, and carcinogen promoter. BMC Cancer 11:232.

unclear at this time, it has been shown that Parg can block Parp1 mediated chromatin remodeling and transcriptional activation in specific circumstances. Therefore, we speculate Parg inhibition might heighten the effects of PARylation, thus promoting the transcription of tumor suppressor genes. Further, in cancer cells having defects in the PARylation pathway such as aberrantly dePARylated Ctcf, Parg inhibition might serve to correct these deficiencies.

Supporting these rationale for Parg inhibition being a novel approach for anti-cancer therapy are several reports indicating Parg inhibition has potent anti-tumor effects against cholangiocarinoma in vivo (Marienfeld et al., 2003), and in vitro data showing growth inhibitory activity of Parg inhibition or knockdown in multiple types of cancer (Fauzee et al., 2012; Feng et al., 2012; Li et al., 2012; Pan et al., 2012). Now that the crystal structure of Parg has been solved (Slade et al., 2011; Kim et al., 2012), there is a clear need to develop specific inhibitors of this enzyme and test their efficacy as anti-cancer therapeutics.

cytotoxicity. Cancer Res. 54, 4627-4634.

Donawho, C. K., Luo, Y., Penning, T. D., Bauch, J. L., Bouska, J. J., BontchevaDiaz, V. D., et al. (2007). ABT-888, an orally active poly(ADP-ribose) polymerase inhibitor that potentiates DNA-damaging agents in preclinical tumor models. Clin. Cancer Res. 13, 2728-2737.

Faraone-Mennella, M. R., De Lucia, F., Palomba, L., Quesada, P., and Farina, B. (1993). Effect of ADPribosylation reaction on bull testis chromatin. Biochem. Mol. Biol. Int. 31, 373-380.

Farrar, D., Rai, S., Chernukhin, I., Jagodic, M., Ito, Y., Yammine, S., et al. (2010). Mutational analysis of the poly(ADP-ribosyl)ation sites of the transcription factor CTCF provides an insight into the mechanism of its regulation by poly(ADPribosyl)ation. Mol. Cell. Biol. 30, 1199-1216.

Fathers, C., Drayton, R. M., Solovieva, S., and Bryant, H. E. (2012). Inhibition of poly(ADP-ribose) glycohydrolase (PARG) specifically kills BRCA2-deficient tumor cells. Cell Cycle 11, 990-997.

Fauzee, N. J., Li, Q., Wang, Y. L., and Pan, J. (2012). Silencing Poly (ADP-Ribose) glycohydrolase (PARG) expression inhibits growth of human colon cancer cells in vitro via PI3K/Akt/NFkappa-B pathway. Pathol. Oncol. Res. 18, 191-199.

Feng, X., Zhou, Y., Proctor, A. M., Hopkins, M. M., Liu, M., and Koh, D. W. (2012). Silencing of apoptosis-inducing factor and poly(ADP-ribose) glycohydrolase reveals novel roles in breast cancer cell death after chemotherapy. Mol. Cancer 11, 48.
Fisher, A. E., Hochegger, H., Takeda, S., and Caldecott, K. W. (2007). Poly(ADP-ribose) polymerase 1 accelerates single-strand break repair in concert with poly(ADPribose) glycohydrolase. Mol. Cell. Biol. 27, 5597-5605.

Fong, P. C., Boss, D. S., Yap, T. A., Tutt, A., Wu, P., Mergui-Roelvink, M., et al. (2009). Inhibition of poly(ADPribose) polymerase in tumors from BRCA mutation carriers. N. Engl. J. Med. 361, 123-134.

Ford, D., Easton, D. F., Stratton, M., Narod, S., Goldgar, D., Devilee, P., et al. (1998). Genetic heterogeneity and penetrance analysis of the BRCA1 and BRCA2 genes in breast cancer families. the breast cancer linkage consortium. Am. J. Hum. Genet. 62, 676-689.

Frizzell, K. M., Gamble, M. J., Berrocal, J. G., Zhang, T., Krishnakumar, R., Cen, Y., et al. (2009). Global analysis of transcriptional regulation by poly(ADP-ribose) polymerase1 and poly(ADP-ribose) glycohydrolase in MCF-7 human breast cancer cells. J. Biol. Chem. 284, 33926-33938.

Gagne, J. P., Isabelle, M., Lo, K. S., Bourassa, S., Hendzel, M. J., Dawson, V. L., et al. (2008). Proteome-wide identification of poly(ADP-ribose) binding proteins and poly(ADP-ribose)-associated protein complexes. Nucleic Acids Res. 36, 6959-6976.

Gagne, J. P., Pic, E., Isabelle, M., Krietsch, J., Ethier, C., Paquet, E., et al. (2012). Quantitative proteomics profiling of the poly(ADPribose)-related response to genotoxic stress. Nucleic Acids Res. 40, 7788-7805. 
Gamble, M. J., and Fisher, R. P. (2007). SET and PARP1 remove DEK from chromatin to permit access by the transcription machinery. Nat. Struct. Mol. Biol. 14, 548-555.

Gelmon, K. A., Tischkowitz, M., Mackay, H., Swenerton, K., Robidoux, A., Tonkin, K., et al. (2011). Olaparib in patients with recurrent highgrade serous or poorly differentiated ovarian carcinoma or triplenegative breast cancer: a phase 2, multicentre, open-label, nonrandomised study. Lancet Oncol. 12, 852-861.

Gonzalez-Angulo, A. M., Timms, K. M., Liu, S., Chen, H., Litton, J. K., Potter, J., et al. (2011). Incidence and outcome of BRCA mutations in unselected patients with triple receptornegative breast cancer. Clin. Cancer Res. 17, 1082-1089.

Gusterson, B. A., Ross, D. T., Heath, V. J., and Stein, T. (2005). Basal cytokeratins and their relationship to the cellular origin and functional classification of breast cancer. Breast Cancer Res. 7, 143-148.

Hang, C. T., Yang, J., Han, P., Cheng, H. L., Shang, C., Ashley, E., et al. (2010). Chromatin regulation by Brg1 underlies heart muscle development and disease. Nature 466, 62-67.

Hassa, P. O., Buerki, C., Lombardi, C., Imhof, R., and Hottiger, M. O. (2003). Transcriptional coactivation of nuclear factor-kappaB-dependent gene expression by $\mathrm{p} 300$ is regulated by poly(ADP)-ribose polymerase- 1 . J. Biol. Chem. 278, 45145-45153.

Helleday, T. (2011). The underlying mechanism for the PARP and BRCA synthetic lethality: clearing up the misunderstandings. Mol. Oncol. 5, 387-393.

Huang, D., Wang, Y., Wang, L., Zhang, F., Deng, S., Wang, R., et al. (2011). Poly(ADP-ribose) polymerase 1 is indispensable for transforming growth factor-beta Induced Smad3 activation in vascular smooth muscle cell. PLOS ONE 6:e27123. doi:10.1371/journal.pone.0027123

Huletsky, A., de Murcia, G., Muller, S., Hengartner, M., Menard, L., Lamarre, D., et al. (1989). The effect of poly(ADP-ribosyl)ation on native and H1-depleted chromatin. A role of poly(ADP-ribosyl)ation on core nucleosome structure. J. Biol. Chem. 264, 8878-8886.

Ju, B. G., Lunyak, V.V., Perissi, V., GarciaBassets, I., Rose, D. W., Glass, C. K., et al. (2006). A topoisomerase IIbetamediated dsDNA break required for regulated transcription. Science 312, 1798-1802.
Ju, B. G., and Rosenfeld, M. G. (2006). A breaking strategy for topoisomerase IIbeta/PARP-1-dependent regulated transcription. Cell Cycle 5, 2557-2560.

Kaur, H., Chen, S., Xin, X., Chiu, J., Khan, Z. A., and Chakrabarti, S. (2006). Diabetes-induced extracellular matrix protein expression is mediated by transcription coactivator p300. Diabetes 55, 3104-3111.

Khan, O. A., Gore, M., Lorigan, P., Stone, J., Greystoke, A., Burke, W., et al. (2011). A phase I study of the safety and tolerability of olaparib (AZD2281, KU0059436) and dacarbazine in patients with advanced solid tumours. Br. J. Cancer 104, 750-755.

Kim, I. K., Kiefer, J. R., Ho, C. M., Stegeman, R. A., Classen, S., Tainer, J. A., et al. (2012). Structure of mammalian poly(ADP-ribose) glycohydrolase reveals a flexible tyrosine clasp as a substrate-binding element. Nat. Struct. Mol. Biol. 19, 653-656.

Kim, M. Y., Mauro, S., Gevry, N., Lis, J. T., and Kraus, W. L. (2004). NAD+-dependent modulation of chromatin structure and transcription by nucleosome binding properties of PARP-1. Cell 119, 803-814.

Kim, M. Y., Zhang, T., and Kraus, W. L. (2005). Poly(ADP-ribosyl)ation by PARP-1: 'PAR-laying' NAD+ into a nuclear signal. Genes Dev. 19, 1951-1967.

Konstantinopoulos, P. A., Spentzos, D., Karlan, B. Y., Taniguchi, T., Fountzilas, E., Francoeur, N., et al. (2010). Gene expression profile of BRCAness that correlates with responsiveness to chemotherapy and with outcome in patients with epithelial ovarian cancer. $J$. Clin. Oncol. 28, 3555-3561.

Krishnakumar, R., Gamble, M. J., Frizzell, K. M., Berrocal, J. G., Kininis, M., and Kraus, W. L. (2008). Reciprocal binding of PARP-1 and histone $\mathrm{H} 1$ at promoters specifies transcriptional outcomes. Science 319, 819-821.

Krishnakumar, R., and Kraus, W. L. (2010). PARP-1 regulates chromatin structure and transcription through a KDM5B-dependent pathway. Mol. Cell 39, 736-749.

Kumari, S. R., Mendoza-Alvarez, H., and Alvarez-Gonzalez, R. (1998). Functional interactions of p53 with poly(ADP-ribose) polymerase (PARP) during apoptosis following DNA damage: covalent poly(ADPribosyl)ation of p53 by exogenous PARP and noncovalent binding of p53 to the $\mathrm{M}(\mathrm{r}) \quad 85,000$ proteolytic fragment. Cancer Res. 58, 5075-5078.

Kummar, S., Chen, A., Ji, J., Zhang, Y., Reid, J. M., Ames, M., et al. (2011). Phase I study of PARP inhibitor ABT-888 in combination with topotecan in adults with refractory solid tumors and lymphomas. Cancer Res. 71, 5626-5634.

Kummar, S., Ji, J., Morgan, R., Lenz, H. J., Puhalla, S. L., Belani, C. P., et al. (2012). A phase I study of veliparib in combination with metronomic cyclophosphamide in adults with refractory solid tumors and lymphomas. Clin. Cancer Res. 18 1726-1734.

Kun, E., Kirsten, E., and Ordahl, C. P. (2002). Coenzymatic activity of randomly broken or intact doublestranded DNAs in auto and histone H1 trans-poly(ADP-ribosylation), catalyzed by poly(ADP-ribose) polymerase (PARP I). J. Biol. Chem. 277, 39066-39069.

Kurian, A. W., Fish, K., Shema, S. J., and Clarke, C. A. (2010). Lifetime risks of specific breast cancer subtypes among women in four racial/ethnic groups. Breast Cancer Res. 12, R99.

Li, M., Naidu, P., Yu, Y., Berger, N. A., and Kannan, P. (2004). Dual regulation of AP-2alpha transcriptional activation by poly(ADPribose) polymerase-1. Biochem. J. 382, 323-329.

Li, Q., Li, M., Wang, Y. L., Fauzee, N. J., Yang, Y., Pan, J., et al. (2012). RNA interference of PARG could inhibit the metastatic potency of colon carcinoma cells via PI3-kinase/Akt pathway. Cell. Physiol. Biochem. 29, 361-372.

Liu, X., Shi, Y., Guan, R., Donawho, C., Luo, Y., Palma, J., et al. (2008). Potentiation of temozolomide cytotoxicity by poly(ADP)ribose polymerase inhibitor ABT-888 requires a conversion of single-stranded DNA damages to double-stranded DNA breaks. Mol. Cancer Res. 6 , 1621-1629.

Liu, X., Shi, Y., Maag, D. X., Palma, J. P., Patterson, M. J., Ellis, P. A., et al. (2012). Iniparib nonselectively modifies cysteine-containing proteins in tumor cells and is not a bona fide PARP inhibitor. Clin. Cancer Res. 18, 510-523.

Lonn, P., van der Heide, L. P., Dahl, M., Hellman, U., Heldin, C. H., and Moustakas, A. (2010). PARP1 attenuates Smad-mediated transcription. Mol. Cell 40, 521-532.

Lonskaya, I., Potaman, V. N., Shlyakhtenko, L. S., Oussatcheva, E. A., Lyubchenko, Y. L., and Soldatenkov, V. A. (2005). Regulation of poly(ADP-ribose) polymerase-1 by DNA structure-specific binding. J. Biol. Chem. 280, 17076-17083.

Lu, H., Wang, X., Li, T., Urvalek, A. M., Yu, L., Li, J., et al. (2011). Identification of poly (ADP-ribose) polymerase-1 (PARP-1) as a novel Kruppel-like factor 8-interacting and -regulating protein. J. Biol. Chem. 286, 20335-20344.

Malanga, M., Pleschke, J. M., Kleczkowska, H. E., and Althaus, F. R. (1998). Poly(ADP-ribose) binds to specific domains of p53 and alters its DNA binding functions. J. Biol. Chem. 273, 11839-11843.

Marienfeld, C., Tadlock, L., Yamagiwa, Y., and Patel, T. (2003). Inhibition of cholangiocarcinoma growth by tannic acid. Hepatology 37, 1097-1104.

Martinez-Zamudio, R., and Ha, H. C. (2012). Histone ADP-ribosylation facilitates gene transcription by directly remodeling nucleosomes. Mol. Cell. Biol. 32, 2490-2502.

Meisterernst, M., Stelzer, G., and Roeder, R. G. (1997). Poly(ADPribose) polymerase enhances activator-dependent transcription in vitro. Proc. Natl. Acad. Sci. U.S.A. 94, 2261-2265.

Mendoza-Alvarez, H., Chavez-Bueno, S., and Alvarez-Gonzalez, R. (2000). Chain length analysis of ADP-ribose polymers generated by poly(ADPribose) polymerase (PARP) as a function of beta-NAD+ and enzyme concentrations. IUBMB Life 50, 145-149.

Mortusewicz, O., Fouquerel, E., Ame, J. C., Leonhardt, H., and Schreiber, V. (2011). PARG is recruited to DNA damage sites through poly(ADPribose)- and PCNA-dependent mechanisms. Nucleic Acids Res. 39, 5045-5056.

Nguyen, D., Zajac-Kaye, M., Rubinstein, L., Voeller, D., Tomaszewski, J. E., Kummar, S., et al. (2011). Poly(ADP-ribose) polymerase inhibition enhances p53-dependent and -independent DNA damage responses induced by DNA damaging agent. Cell Cycle 10, 4074-4082.

Ogino, H., Nozaki, T., Gunji, A., Maeda, M., Suzuki, H., Ohta, T., et al. (2007) Loss of Parp-1 affects gene expression profile in a genome-wide manner in ES cells and liver cells. BMC Genomics 8:41. doi:10.1186/14712164-8-41

Okada, H., Inoue, T., Kikuta, T., Kato, N., Kanno, Y., Hirosawa, N., et al. (2008). Poly(ADP-ribose) polymerase-1 enhances transcription of the profibrotic CCN2 gene. J. Am. Soc. Nephrol. 19, 933-942. 
Olabisi, O. A., Soto-Nieves, N., Nieves, E., Yang, T. T., Yang, X., Yu, R. Y., et al. (2008). Regulation of transcription factor NFAT by ADP-ribosylation. Mol. Cell. Biol. 28, 2860-2871.

Oonk, A. M., van Rijn, C., Smits, M. M., Mulder, L., Laddach, N., Savola, S. P., et al. (2012). Clinical correlates of 'BRCAness' in triple-negative breast cancer of patients receiving adjuvant chemotherapy. Ann. Oncol. 23, 2301-2305.

O'Shaughnessy, J. (2011). A randomized phase III study of iniparib (BSI-201) in combination with gemcitabine/carboplatin (G/C) in metastatic triple-negative breast cancer (TNBC). J. Clin. Oncol. 29, abstr. 1007. [ASCO meeting abstracts].

O'Shaughnessy, J., Osborne, C., Pippen, J., Yoffe, D., Patt, G., Monaghan, C., et al. (2009). Efficacy of BSI-201, a poly (ADP-ribose) polymerase-1 (PARP1) inhibitor, in combination with gemcitabine/carboplatin (G/C) in patients with metastatic triplenegative breast cancer (TNBC): results of a randomized phase II trial. J. Clin. Oncol. 27, abstr. 3. [ASCO meeting abstracts].

O'Shaughnessy, J., Osborne, C., Pippen, J. E., Yoffe, M., Patt, D., Rocha, C., et al. (2011). Iniparib plus chemotherapy in metastatic triple-negative breast cancer. N. Engl. J. Med. 364, 205-214.

Pan, J., Fauzee, N. J., Wang, Y. L., Sheng, Y. T., Tang, Y., Wang, J. Q., et al. (2012). Effect of silencing PARG in human colon carcinoma LoVo cells on the ability of HUVEC migration and proliferation. Cancer Gene Ther. 19, 715-722.

Pant, V., Kurukuti, S., Pugacheva, E., Shamsuddin, S., Mariano, P., Renkawitz, R., et al. (2004). Mutation of a single CTCF target site within the H19 imprinting control region leads to loss of Igf2 imprinting and complex patterns of de novo methylation upon maternal inheritance. Mol. Cell. Biol. 24, 3497-3504.

Patel, A. G., De Lorenzo, S. B., Flatten, K. S., Poirier, G. G., and Kaufmann, S. H. (2012). Failure of iniparib to inhibit poly(ADP-Ribose) polymerase in vitro. Clin. Cancer Res. 18, 1655-1662.

Pavri, R., Lewis, B., Kim, T. K., Dilworth, F. J., Erdjument-Bromage, H., Tempst, P., et al. (2005). PARP-1 determines specificity in a retinoid signaling pathway via direct modulation of mediator. Mol. Cell 18, 83-96.

Pern, F., Bogdanova, N., Schurmann, P., Lin, M., Ay, A., Langer, F., et al. (2012). Mutation analysis of
BRCA1, BRCA2, PALB2 and BRD7 in a hospital-based series of German patients with triple-negative breast cancer. PLoS ONE 7:e47993. doi:10.1371/journal.pone.0047993

Petesch, S. J., and Lis, J. T. (2008). Rapid, transcription-independent loss of nucleosomes over a large chromatin domain at Hsp70 loci. Cell 134, 74-84.

Phuah, S. Y., Looi, L. M., Hassan, N., Rhodes, A., Dean, S., Mohd Taib, N. A., et al. (2012). Triple negative breast cancer and phosphatase and tensin homolog loss are predictors of BRCAl germline mutations in women with early onset and familial breast cancer, but not in women with isolated late-onset breast cancer. Breast Cancer Res. 14, R142.

Pinnola, A., Naumova, N., Shah, M., and Tulin, A. V. (2007). Nucleosomal core histones mediate dynamic regulation of poly(ADP-ribose) polymerase 1 protein binding to chromatin and induction of its enzymatic activity. J. Biol. Chem. 282, 32511-32519.

Plummer, R., Jones, C., Middleton, M., Wilson, R., Evans, J., Olsen, A., et al. (2008). Phase I study of the poly(ADP-ribose) polymerase inhibitor, AG014699, in combination with temozolomide in patients with advanced solid tumors. Clin. Cancer Res. 14, 7917-7923.

Poirier, G. G., de Murcia, G., JongstraBilen, J., Niedergang, C., and Mandel, P. (1982). Poly(ADPribosyl)ation of polynucleosomes causes relaxation of chromatin structure. Proc. Natl. Acad. Sci. U.S.A. 79, 3423-3427.

Prat, A., Parker, J. S., Karginova, O., Fan, C., Livasy, C., Herschkowitz, J. I., et al. (2010). Phenotypic and molecular characterization of the claudinlow intrinsic subtype of breast cancer. Breast Cancer Res. 12, R68.

Rajan, A., Carter, C. A., Kelly, R. J., Gutierrez, M., Kummar, S., Szabo, E., et al. (2012). A phase I combination study of olaparib with cisplatin and gemcitabine in adults with solid tumors. Clin. Cancer Res. 18, 2344-2351.

Ratner, E. S., Sartorelli, A. C., and Lin, Z. P. (2012). Poly (ADP-ribose) polymerase inhibitors: on the horizon of tailored and personalized therapies for epithelial ovarian cancer. Curr. Opin. Oncol. 24, 564-571.

Reale, A., Matteis, G. D., Galleazzi, G., Zampieri, M., and Caiafa, P. (2005). Modulation of DNMT1 activity by ADP-ribose polymers. Oncogene 24, 13-19.

Sala, A., La Rocca, G., Burgio, G., Kotova, E., Di Gesu, D.,
Collesano, M., et al. (2008). The nucleosome-remodeling ATPase ISWI is regulated by poly-ADPribosylation. PLoS Biol. 6:e252. doi:10.1371/journal.pbio.0060252

Santilli, G., Cervellera, M. N., Johnson, T. K., Lewis, R. E., Iacobelli, S., and Sala, A. (2001). PARP co-activates BMYB through enhanced phosphorylation at cyclin/cdk2 sites. Oncogene 20, 8167-8174.

Slade, D., Dunstan, M. S., Barkauskaite, E., Weston, R., Lafite, P., Dixon, N., et al. (2011). The structure and catalytic mechanism of a poly(ADPribose) glycohydrolase. Nature 477, 616-620.

Slattery, E., Dignam, J. D., Matsui, T., and Roeder, R. G. (1983). Purification and analysis of a factor which suppresses nick-induced transcription by RNA polymerase II and its identity with poly(ADP-ribose) polymerase. J. Biol. Chem. 258 5955-5959.

Talbert, P. B., and Henikoff, S. (2006). Spreading of silent chromatin: inaction at a distance. Nat. Rev. Genet. 7, 793-803.

Tulin, A., and Spradling, A. (2003). Chromatin loosening by poly(ADP)-ribose polymerase (PARP) at Drosophila puff loci. Science 299, 560-562.

Turner, N., Tutt, A., and Ashworth, A. (2004). Hallmarks of 'BRCAness' in sporadic cancers. Nat. Rev. Cancer 4 , 814-819.

Tutt, A., Robson, M., Garber, J. E., Domchek, S. M., Audeh, M. W., Weitzel, J. N., et al. (2010). Oral poly(ADPribose) polymerase inhibitor olaparib in patients with BRCA1 or BRCA2 mutations and advanced breast cancer: a proof-of-concept trial. Lancet 376, 235-244.

Wacker, D. A., Ruhl, D. D., Balagamwala, E. H., Hope, K. M., Zhang, T., and Kraus, W. L. (2007). The DNA binding and catalytic domains of poly(ADP-ribose) polymerase 1 cooperate in the regulation of chromatin structure and transcription. Mol. Cell. Biol. 27, 7475-7485.

Witcher, M., and Emerson, B. M. (2009). Epigenetic silencing of the p16(INK4a) tumor suppressor is associated with loss of CTCF binding and a chromatin boundary. Mol. Cell 34, 271-284.

Yu, W., Ginjala, V., Pant, V., Chernukhin, I., Whitehead, J., Docquier, F., et al. (2004). Poly(ADP-ribosyl)ation regulates CTCF-dependent chromatin insulation. Nat. Genet. 36, 1105-1110.

Zampieri, M., Guastafierro, T., Calabrese, R., Ciccarone, F., Bacalini, M. G., Reale, A., et al. (2012).
ADP-ribose polymers localized on Ctcf-Parp1-Dnmtl complex prevent methylation of Ctcf target sites. Biochem. J. 441, 645-652.

Zampieri, M., Passananti, C., Calabrese, R., Perilli, M., Corbi, N., De Cave, F., et al. (2009). Parp1 localizes within the Dnmtl promoter and protects its unmethylated state by its enzymatic activity. PLoS ONE 4:e4717. doi:10.1371/journal.pone.0004717

Zaniolo, K., Desnoyers, S., Leclerc, S., and Guerin, S. L. (2007). Regulation of poly(ADP-ribose) polymerase-1 (PARP-1) gene expression through the post-translational modification of Spl: a nuclear target protein of PARP-1. BMC Mol. Biol. 8:96. doi:10.1186/1471-2199-8-96

Zhang, T., Berrocal, J. G., Yao, J., DuMond, M. E., Krishnakumar, R., Ruhl, D. D., et al. (2012). Regulation of poly(ADP-ribose) polymerase-1-dependent gene expression through promoterdirected recruitment of a nuclear NAD+ synthase. J. Biol. Chem. 287, 12405-12416.

Ziegler, M., and Oei, S. L. (2001). A cellular survival switch: poly(ADPribosyl)ation stimulates DNA repair and silences transcription. Bioessays 23, 543-548.

Zobeck, K. L., Buckley, M. S., Zipfel, W. R., and Lis, J. T. (2010). Recruitment timing and dynamics of transcription factors at the Hsp70 loci in living cells. Mol. Cell 40, 965-975.

Conflict of Interest Statement: The authors declare that the research was conducted in the absence of any commercial or financial relationships that could be construed as a potential conflict of interest.

Received: 11 October 2012; paper pending published: 01 November 2012; accepted: 06 December 2012; published online: 27 December 2012.

Citation: Lovato A, Panasci $L$ and Witcher M (2012) Is there an epigenetic component underlying the resistance of triple-negative breast cancers to Parp inhibitors? Front. Pharmacol. 3:202. doi: 10.3389/fphar.2012.00202

This article was submitted to Frontiers in Pharmacology of Anti-Cancer Drugs, a specialty of Frontiers in Pharmacology. Copyright $\odot 2012$ Lovato, Panasci and Witcher. This is an open-access article distributed under the terms of the Creative Commons Attribution License, which permits use, distribution and reproduction in other forums, provided the original authors and source are credited and subject to any copyright notices concerning any third-party graphics etc. 